\title{
Construction of a Statistical Surgical Plan Atlas for Automated 3D Planning of Femoral Component in Total Hip Arthroplasty
}

\author{
Masahiko Nakamoto ${ }^{1}$, Itaru Otomaru ${ }^{2}$, Masaki Takao ${ }^{3}$, Nobuhiko Sugano $^{3}$, \\ Yoshiyuki Kagiyama ${ }^{4}$, Hideki Yoshikawa ${ }^{3}$, Yukio Tada ${ }^{2}$, and Yoshinobu Sato ${ }^{1}$ \\ ${ }^{1}$ Division of Image Analysis, Graduate School of Medicine, Osaka University, Japan \\ ${ }^{2}$ Graduate School of Engineering, Kobe University, Japan \\ ${ }^{3}$ Department of Orthopaedics, Graduate School of Medicine, Osaka University, Japan \\ ${ }^{4}$ The Center for Advanced Medical Engineering and Informatics, \\ Osaka University, Japan
}

\begin{abstract}
The problem of automating preoperative planning of the femoral component (stem) for total hip arthroplasty (THA) is addressed. In our previous method, time-consuming trial-and-error processes were involved in parameter tuning of the objective function. This problem prevents application in different stem systems. To overcome this problem, a statistical surgical plan atlas (SSPA) is constructed from training datasets of stem planning. The SSPA represents the average and variance of the distance distribution on the stem surface to the femoral canal surface. That is, it encodes the distribution of the degree of contact preferred by the surgeon. Automated planning is performed by minimizing the squared difference between distributions of the SSPA and planning solution. The proposed method involves no parameter tuning to define the objective function that evaluates differences from the planning the surgeon prefers. Experimental evaluations showed that the proposed method renders parameter tuning unnecessary while it still provides comparable accuracy to the previous method.
\end{abstract}

\section{Introduction}

Preoperative 3D planning is becoming important, because the preoperative 3D plan can be executed exactly using recent intraoperative navigation and robotic systems, particularly in orthopedics. Since total hip arthroplasty is one of bestfit procedures, commercial systems are gaining wide use and clinical experiences of their application have been reported [1] 2]. Intraoperative assistance systems have thus been developed to place hip implants accurately according to the preoperative plan 3, 4] 5. When these systems are used for THA, preoperative $3 \mathrm{D}$ planning is essential and crucial for surgical outcomes. The present paper addresses preoperative planning for the femoral component (stem) of the hip implants. Currently, the surgeon determines position, orientation and size of the stem using interactive planning software [6] so that the stem is stably fixed to 
the host bone, that is, the femoral canal. Such 3D planning still depends on interactive operations of the surgeon, and thus is subjective and time-consuming. Although a measure of stem fitness has been used as an objective criterion 7] 8] 9], no planning procedure using this measure has yet been established. To overcome these problems, an automated preoperative planning system for THA has been developed to automatically determine optimal size, position and orientation of the stem, which maximizes the objective function representing stem fitness [10] 11. Although the developed system has found useful, time-consuming trial-and-error processes were required to adjust the various parameters involved in the objective function. These processes are needed for each different stem design, preventing ready application of the method for various stem designs provided by different manufacturers.

We propose herein a statistical surgical plan atlas (SSPA) to overcome this problem. The SSPA is constructed from training datasets of stem planning made by an experienced surgeon. The SSPA represents the average and variance of the distance distribution from the stem surface to the femoral canal surface. That is, it encodes the distribution of the degree of contact preferred by the surgeon. The problem of determining the optimal plan realizing good stem fitness is formulated as a matching problem with the SSPA. In the proposed method, no parameter tuning is required for the objective function. Viceconti et al. also proposed an automated planning method based on the surgeon's planning [12. While Viceconti's method depends on the plan of a single surgeon, the proposed method is based on a number of plans to define the objective function representing stem fitness. We applied the proposed method to 18 cases and compared the planning results with those of an experienced surgeon.

\section{Methods}

\subsection{Overview of Automated Planning of Stem Placement}

Fig. 1]shows an overview of the proposed system. For automated planning of stem placement, we assume that 3D surface models of the femoral canal reconstructed from preoperative CT by manual segmentation and all variations of the stems are available. We also assume that a number of stem placement plans made by an experienced surgeon are available. The proposed method determines the position and orientation of the stem $T$, which is a $4 \times 4$ rigid transformation matrix, and the size of the stem $s$ from the above described input datasets.

\subsection{Construction of Statistical Surgical Plan Atlas}

A stem has several size variations to adapt to various sizes of femoral bones in practice. To integrate distributions of various stem sizes into one SSPA, correspondence among stems of different sizes by nonrigid registration is required because stem shapes differ slightly and are not similar for each size. A stem of a certain size is determined as a reference stem, then correspondences between surface models of the reference stem and other stems are made by point-based 
nonrigid registration [13]. Using the obtained correspondence, distance distributions of stems other than the reference stem are mapped to the reference stem, thus constructing the SSPA from the plans of various stem sizes.

Let $d(\mathbf{p}, Q)$ be a signed distance between a point on the stem surface used for planning and the femoral canal surface, where $\mathbf{p}=(x, y, z)$ is a position on the stem surface and $Q$ is a 3D surface model of the femoral canal. If a gap exists between a point and the canal surface, then $d(\mathbf{p}, Q)$ is positive, and if the point penetrates the canal surface, the value is negative. When surface models of a stem and femoral canal are given with relative position and orientation, as a plan of stem placement, distance is calculated for each vertex of the stem model, and the distance distribution can thus be obtained. The SSPA consists of the average and standard deviation (SD) of the distance distribution. If $n$ datasets of the surgical plan are given, an average of the distance distribution $U$ and $\mathrm{SD}$ of the distribution $S$ are defined as

$$
\begin{array}{r}
U=\left\{\mu_{i} \mid \mu_{i}=\frac{1}{n} \sum_{j=1}^{n} d\left(\mathbf{p}_{j}, Q\right), \mathbf{p}_{i} \in V\right\} \\
S=\left\{\sigma_{i} \mid \sigma_{i}=\sqrt{\frac{1}{n} \sum_{j=1}^{n}\left(d\left(\mathbf{p}_{j}, Q\right)-\mu_{j}\right)^{2}}, \mathbf{p}_{i} \in V\right\},
\end{array}
$$

where $V$ is the set of vertex positions of the stem model, and $\mu_{i}$ and $\sigma_{i}$ represent average and SD of the distance distribution at the point $\mathbf{p}_{i}$, respectively.

\subsection{Optimization Procedure}

A rigid transformation matrix $T$, which means position and orientation of the stem, and size $s$ are determined by minimizing the cost function $f(T, s)$ representing difference of distance distributions between the SSPA and planning solution. The cost function $f(T, s)$ is defined as

$$
f(T, s)=\sum_{i=1}^{m} \frac{1}{\sigma_{i}^{2}}\left(d\left(T \mathbf{p}_{i s}, Q\right)-\mu_{i}\right)^{2}
$$

where $m$ is the number of vertices on the reference stem surface model, and $\mathbf{p}_{i s}$ is $i$-th vertex position of the stem surface model of size $s$. Minimization of the cost function is performed by two steps. First, the optimal position and orientation $\hat{T}_{k}$ for the stem of size $k$ is obtained by $\hat{T}_{k}=\underset{T}{\arg \min } f(T, k)$ for each size of the stem. Next, the optimal size $\hat{s}$ is obtained by $\hat{s}=\underset{k=\left\{k_{1}, \cdots, k_{l}\right\}}{\arg \min }\left\{f\left(\hat{T}_{k_{1}}, k_{1}\right), \cdots, f\left(\hat{T}_{k_{l}}, k_{l}\right)\right\}$, where $l$ is the number of the stem size variation.

To obtain the solution close to the global minimum as much as possible, multiple minimization processes are performed from a number of initial positions and orientations within a reasonable range. Minimization of the cost function was performed using the Powell method. Let $\mathbf{t}_{0}$ and $\mathbf{r}_{0}$ be centers of the range for initial positions and orientations, respectively. $\mathbf{t}_{0}$ was defined as the position 
where the origin of the stem coordinate system was on the $z$-axis (i.e. long axis) of the femoral coordinate system and the $z$-position of the tip of the stem neck was same as that of the neck saddle of the femur. $\mathbf{r}_{0}$ was defined as the direction of the long axis of the stem was same as that of the femoral coordinate system and the anteversion angle (i.e. the angle around the long axis) of the stem was same as that of the femur. Initial position varied from $-8.0 \mathrm{~mm}$ to $8.0 \mathrm{~mm}$ around the position $\mathbf{t}_{0}$ at intervals of $4.0 \mathrm{~mm}$ for each direction. Initial orientation varied from $-4.0 \mathrm{deg}$. to $4.0 \mathrm{deg}$. around the orientation $\mathbf{r}_{0}$ at intervals of $4.0 \mathrm{deg}$. for each angle. We performed preliminary experiments to investigate appropriate intervals of the initial position and orientation. Since no significant differences ( $0.3 \mathrm{~mm}$ in position and $0.2 \mathrm{deg}$. in orientation) existed between the case of 1.0 $\mathrm{mm}$ and $1.0 \mathrm{deg}$., and $4.0 \mathrm{~mm}$ and $4.0 \mathrm{deg}$., the intervals were set to $4.0 \mathrm{~mm}$ and $4.0 \mathrm{deg}$.

\section{Experiments}

\subsection{Experimental Conditions}

We evaluated both the previous method described in [1] and the proposed method, then compared results. CT slice thickness and reconstruction pitch were $2 \mathrm{~mm}$ at the proximal part of the femur and $6 \mathrm{~mm}$ at the distal part of the femur. Field of view $($ FOV) was $360 \times 360 \mathrm{~mm}$. The femoral canal surface was

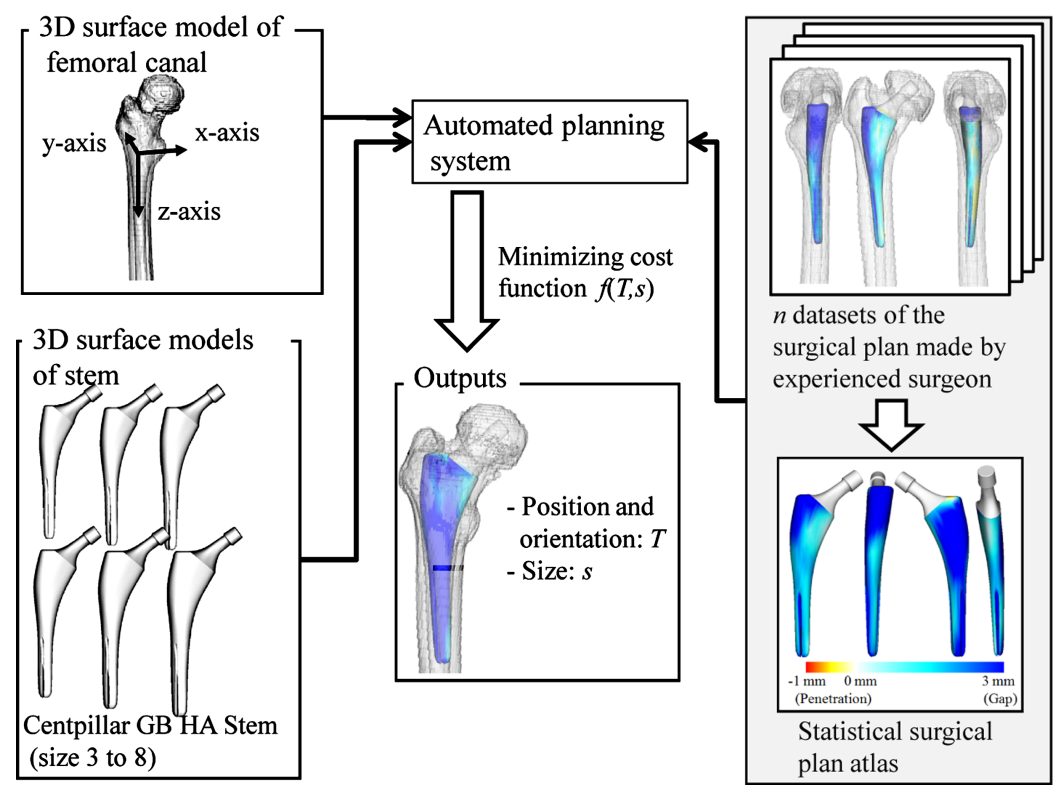

Fig. 1. Block diagram of proposed system 
segmented with a threshold of 800 Hounsfield units (HU). As stem product, Centpillar GB HA stems (Stryker Orthopaedics, Mahwah, NJ, USA) of size 3 to 8 were used (left side of Fig. 1). Stem for size 5 was used as the reference stem to construct the SSPA. We employed 18 cases, plans for which were made by experienced surgeons using the Stryker CT-Hip System (Stryker Leibinger GmbH, Freiburg, Germany). In these cases, surgery was performed with guidance from a surgical navigation system based on the plan.

Evaluation was performed using a leave-one-out cross validation method. The SSPA was constructed from 17 cases, then the remaining 1 case was used for evaluation. Planning results from the automated method were compared with that by the experienced surgeon. Differences in position, orientation and size between plans were calculated. Position and orientation of a solution of the same size as the plan by the surgeon were compared with those from the surgeon's plan, as position and orientation change when size changes and a difference of one size is usually acceptable.

\subsection{Results}

The obtained SSPA is shown in Fig. 2. Color on the stem surface indicates distribution of the average and SD of the distance. The color bar in the bottom of the figure shows the value for each color. Small average and SD were observed in the upper medial and middle lateral areas of the stem, which are shown as pale cyan in Fig. 2, Results of comparison are shown in Fig. 3 and 4 . With the previous method, mean differences in position and orientation were $3.9 \mathrm{~mm}$ and $4.9 \mathrm{deg}$., respectively, and stem sizes matched the plan of the surgeon in 10 cases. With the proposed method, mean differences in position and orientation were

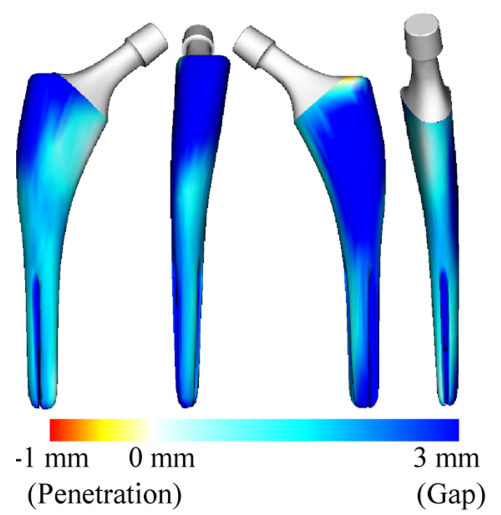

(a)

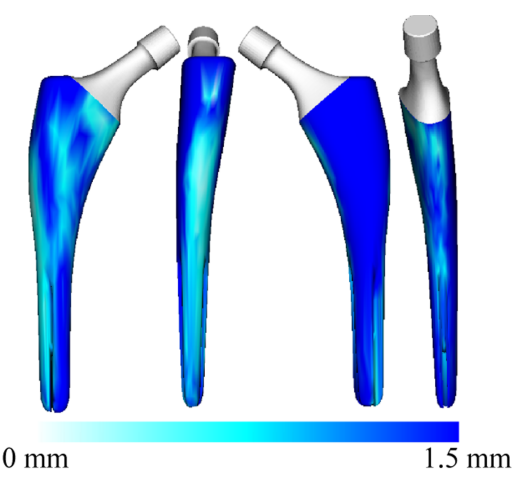

(b)

Fig. 2. Statistical surgical plan atlas. Colors on stem surface indicate average and standard deviation of the distribution of distance from stem surface to femoral canal. Color bar shows the value for each color. (a) Average of distance distribution. (b) Standard deviation of distance distribution. 


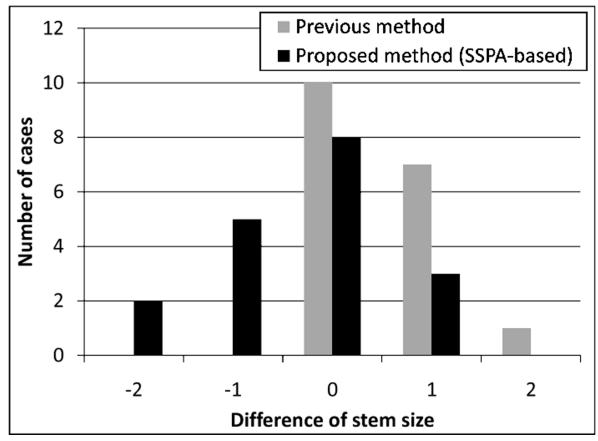

Fig. 3. Differences in stem size between automated planning results and surgeon plans

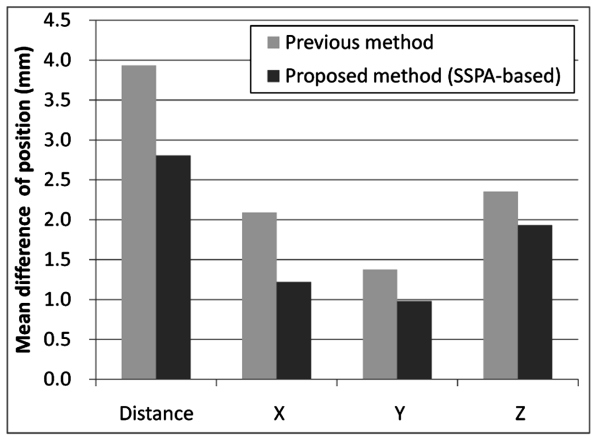

(a) Mean difference in stem position.

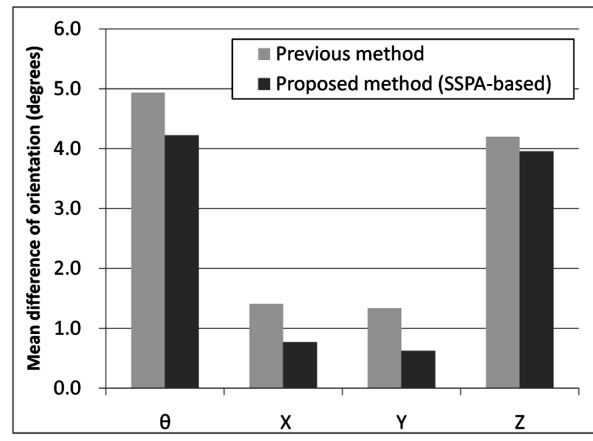

(b) Mean difference in stem orientation. $\theta$ is mean difference angle in the axis-angle representation.

Fig. 4. Results of comparison between automated planning results and surgeon plans

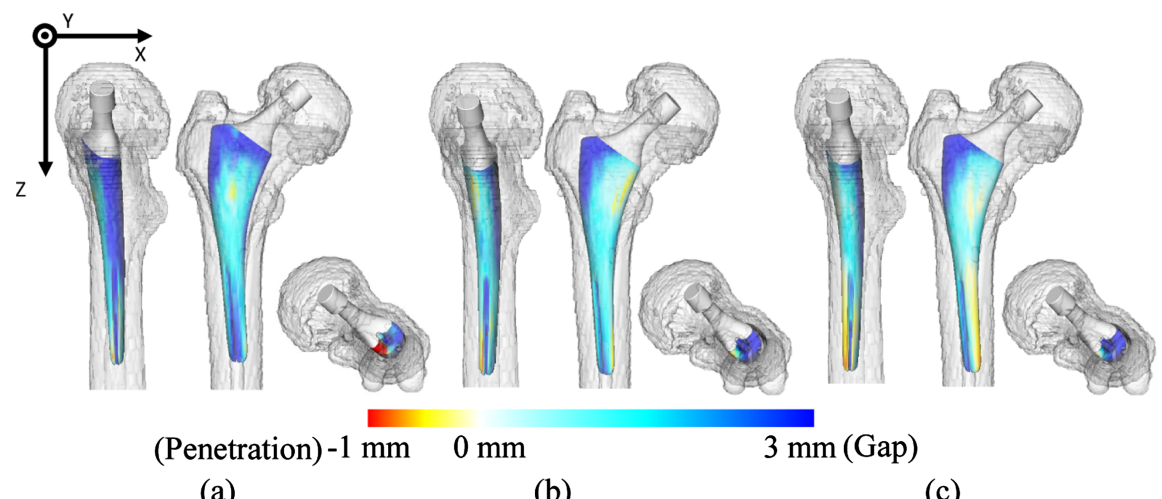

(a)

(b)

(c)

Fig. 5. Visualization of each planning result. Colors on stem surface indicate distance distribution. Color bar shows value for each color. (a) Previous method. (b) Proposed method (SSPA-based) (c) Experienced surgeon. 
$2.8 \mathrm{~mm}$ and $4.2 \mathrm{deg}$., respectively, and stem sizes matched plan of the surgeon in 8 cases. Mean differences in position and orientation were slightly better for the proposed method than for the previous method. The planning result of the typical case is shown in Fig. 5. Colors on the stem surface indicate distribution of the distance. In this case, position along the $z$-axis of the previous method different greatly from the plan of the surgeon. The distribution of the proposed method in the upper medial area resembled that of the surgeon's plan.

\section{Discussion and Conclusions}

We proposed an automated 3D planning method for stem placement in THA based on the SSPA. Experimental results showed that mean differences in position and orientation were better for the proposed method than for the previous method (Fig. 4).

The proposed method assumes that the surgeon makes a plan using a consistent policy. Areas in which the average and SD of distance distributions were small were observed, as shown in Fig. 2 and similar distance distributions in those areas of the planning result were obtained, as shown in Fig. 5] (b) and (c). These results demonstrate that the plans used as training datasets satisfied the assumption and that the SSPA was effective in obtaining a plan reflecting the preferences of the surgeon. We therefore consider that the proposed method would work well even for a different kind of stem if a number of plans were made by the surgeon using a consistent policy. In summary, the significance findings of this study can be summarized as follows:

- The proposed method renders parameter tuning unnecessary, while it still provides comparable accuracy to the previous method.

- The proposed method provides a framework for learning and emulating the planning policy of the surgeon which would be potentially applicable to the stems of various designs.

Stem size selection of the proposed method was slightly inferior to that of the previous method. Fig. 3 shows that the previous method did not select any smallsized stems, while the proposed method selected some small-sized stems. This would be because the previous method of selecting a large-sized stem as long as penetration does not occur between the stem and femoral canal is effective. We consider that the proposed method needs another algorithm to improve size selection, as the cost function is not very sensitive to changes in stem size.

As future work, we will introduce a constraint to prohibit penetration of the stem with the whole stem surface. Since this is a fundamental constraint for making a surgical plan, we consider that this constraint would be incorporated into the optimization procedure independently of the SSPA. This constraint does not involve any parameters, and thus it can be incorporated into the proposed method without spoiling the advantage of the proposed method. 


\section{References}

1. Honl, M., Dierk, O., Gauck, C., et al.: Comparison of robotic-assisted and manual implantation of a primary total hip replacement: A prospective study. Journal of Bone and Joint Surgery - Series A 85(8), 1470-1478 (2003)

2. Ecker, T.M., Tannast, M., Murphy, S.: Computed tomography-based surgical navigation for hip arthroplasty. Clinical Orthopaedics and Related Research 465, 100105 (2007)

3. Bargar, W.L., Bauer, A., Borner, M.: Primary and revision total hip replacement using the Robodoc system. Clinical Orthopaedics and Related Research 354, 82-91 (1998)

4. Sugano, N.: Computer-assisted orthopedic surgery. Journal of Orthopaedic Science 8, 442-448 (2003)

5. Nishihara, S., Sugano, N., Nishii, T., et al.: Comparison between hand rasping and robotic milling for stem implantation in cementless total hip arthroplasty. The Journal of Arthroplasty 21, 957-966 (2006)

6. Lattanzi, R., Viceconti, M., Zannoni, C., et al.: Hip-Op: an innovative software to plan total hip replacement surgery. Medical Informatics and the Internet in Medicine 27, 71-83 (2002)

7. Laine, H., Puolakka, T.J.S., Moilanen, T., et al.: others,: The effects of cementless femoral stem shape and proximal surface texture on 'fit-and-fill' characteristics and on bone remodeling. International Orthopaedics 24(4), 184-190 (2000)

8. Nishihara, S., Sugano, N., Nishii, T., et al.: Comparison of the fit and fill between the anatomic hip femoral component and the VerSys taper femoral component using virtual implantation on the ORTHODOC workstation. Journal of Orthopaedic Science 8, 352-360 (2003)

9. Howard, J.L., Hui, A.J., Bourne, R.B., et al.: A quantitative analysis of bone support comparing cementless tapered and distal fixation total hip replacements. The Journal of Arthroplasty 19, 266-273 (2004)

10. Nakamoto, M., Sato, Y., Sugano, N., et al.: Automated CT-based 3D surgical planning for total hip replacement: a pilot study. In: International Congress Series (2003)

11. Otomaru, I., Takao, M., Nakamoto, M., et al.: Automated preoperative planning system of total hip arthroplasty using anatomical femoral components. In: 6th Annual Meeting of CAOS-International Proceedings (2006)

12. Viceconti, M., Testi, D., Simeoni, M., et al.: An automated method to position prosthetic components within multiple anatomical spaces. Computer Methods and Programs in Biomedicine 70(2), 121-127 (2003)

13. Chui, H., Rangarajan, A.: A new point matching algorithm for non-rigid registration. Computer Vision and Image Understanding 89, 114-141 (2003) 